\title{
Temperature Fluctuations in Homogeneous Turbulence at Four Point Correlations with Variable Prandtl Number
}

\author{
A. Malek ${ }^{1}$, M. A. K. Azad ${ }^{2}$ and M. A. Bkar Pk ${ }^{3}$, \\ ${ }^{I}$ (Department of Applied Mathematics, University of Rajshahi, Rajshahi, Bangladesh) \\ ${ }_{2}^{2}$ (Department of Applied Mathematics, University of Rajshahi, Rajshahi, Bangladesh) \\ 3(Department of Applied Mathematics, University of Rajshahi, Rajshahi, Bangladesh)
}

\begin{abstract}
In this study the decay of temperature fluctuations in homogeneous turbulence before the final period is analyzed by using the correlation equations for fluctuating quantities at four point in the flow field. Throughout this work three- and four point-correlation equations are obtained. The correlation equations are converted into spectral form by their Fourier-transform. The set of equations are made to determinate by neglecting the quintuple correlations in comparison to the fourth-order correlation terms. Finally by integration of the energy spectrum over all wave numbers, we have obtained the decay of energy of temperature fluctuations for four point correlations. The obtained results have been shown by graphically at different Prandtl No. and at the different state of temperature. We also determined the values of the constant appear at the energy equation (38) by using the values of the parameters existing in it for different fluids and the effects of the parameters have been tried to shown by graphically.
\end{abstract}

Keywords- Deissler's method, Four-point correlation, Decay before the temperature fluctuations, final period.

\section{Introduction}

The homogeneous turbulence problem is generated by first specifying the multipoint velocity correlations or their spectral equivalents at an initial time. Those quantities, together with the correlation or spectral equations, are then used to calculate initial time derivatives of correlations or spectra. The derivatives in turn are used in time series to calculate the evolution of turbulence quantities with time. When the problem is treated in this way, the correlation equations are closed by the initial specification of the turbulence and no closure assumption is necessary. An exponential series which is an iterative solution of the Navier stokes equations gave much better results than a Taylor power series when used with the limited available initial data. In the past a remarkable works have been done by some researchers. Taylor introduced correlation coefficients between the fluctuating quantities. Taylor [1] also defined correlation coefficients based on the Eulerian view, which involves the value of the fluctuating-quantity at two points in space. Chandrasekhar [2] studied the invariant theory of isotropic turbulence in magneto-hydrodynamics. Corrsin [3] considered on spectrum of isotropic temperature fluctuations in isotropic turbulence. Deissler $[4,5]$ developed a theory on decay of homogeneous turbulence for times before the final period for three and four point correlation. In the next, Loeffler and Deissler [6] extended their theory for the case of decaying of temperature fluctuations in homogeneous turbulence. Recently, following Deissler [4, 5], Sarker and Azad [7] studied the decay of temperature fluctuations in homogeneous turbulence before the final period for the case of multi-point and multi-time in a rotating system. Azad and Sarker [8,9,10], Azad et al [11] have been done their work on decay of temperature fluctuations in homogeneous and MHD turbulence before the final period for the case of multipoint and multi-time considering dust particles and Coriolis force. In recent times, H. U. Molla et al [12], Azad and Mumtahinah $[14,16]$ have done their research on decaying of energy of temperature fluctuations for the case of dusty fluid homogeneous turbulence due to the effect of Coriolis force for three point correlations. Bkar PK et al [13] calculated the decay of energy of MHD turbulence for four-point correlation. Bkar PK et al [15] studied the homogeneous turbulence in a first-order reactant for the case of multi-point and multi-time prior to the final period of decay in a rotating system. The above researchers have considered two and three point correlation equations and solved these equations after neglecting the fourth and higher order correlation terms.

The main purpose of the present study is to find a possible solution for the dynamics of decaying the temperature fluctuation in homogeneous turbulence for four point correlation. Actually homogeneous turbulence can be produced, for instance, by passing a fluid through a grid, various stages in the decay process then occur various distances downstream from the grid. Through this study, using Deissler's $(1958,1960)$ method we have obtained the decay of temperature fluctuations in homogeneous turbulence at times before the final period for four- point correlation system. The decay law comes out in the following form

$\left\langle T^{2}\right\rangle=W\left(t-t_{0}\right)^{-3 / 2}+X\left(t-t_{0}\right)^{-5}+Y\left(t-t_{1}\right)^{-7 / 2}+Z\left(t-t_{1}\right)^{-7}$, Where $\left\langle T^{2}\right\rangle$ denotes the total energy and $t$ is the time, $\mathrm{W}, \mathrm{X}, \mathrm{Y}$ and $\mathrm{Z}$ are arbitrary constants determined by initial conditions. 


\section{Correlation and Spectral equations}

In order to find the four point correlations and spectral equations for single time and four point correlation we take the momentum equation of turbulence at the point $\mathrm{P}$ and the energy equation of Temperature fluctuation for four point correlations at $P^{\prime}, P^{\prime \prime}$ and $P^{\prime \prime \prime}$ with position vectors $\hat{r}, \hat{r}^{\prime}$ and $\hat{r}^{\prime \prime}$

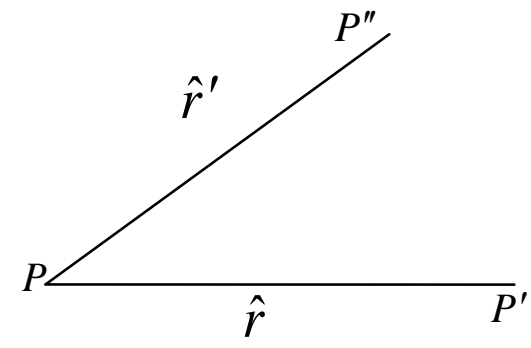

Fig-1:

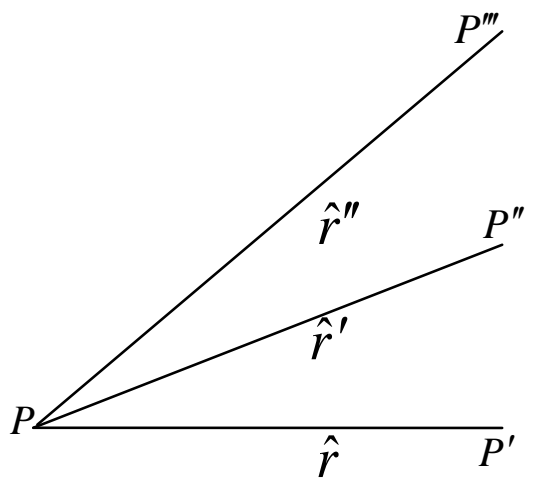

Fig-2:

Fig-1 and: Fig-2:Represent vector configuration for three and four point correlation

$$
\begin{aligned}
& \frac{\partial u_{j}}{\partial t}+\frac{\partial\left(u_{j} u_{i}\right)}{\partial x_{i}}=-\frac{1}{\rho} \frac{\partial P}{\partial x_{j}}+\frac{v}{P_{r}} \frac{\partial^{2} u_{j}}{\partial x_{i} \partial x_{i}} \\
& \frac{\partial \boldsymbol{T}_{i}^{\prime}}{\partial t}+u_{i}^{\prime} \frac{\partial \boldsymbol{T}_{i}^{\prime}}{\partial x_{i}^{\prime}}=\frac{v}{p_{r}} \frac{\partial^{2} \boldsymbol{T}_{i}^{\prime}}{\partial x_{i}^{\prime} \partial x_{i}^{\prime}} \\
& \frac{\partial T_{j}^{\prime \prime}}{\partial t}+u_{i}^{\prime \prime} \frac{\partial T_{j}^{\prime \prime}}{\partial x_{i}^{\prime \prime}}=\frac{v}{p_{r}} \frac{\partial^{2} T_{j}^{\prime \prime}}{\partial x_{i}^{\prime \prime} x_{i}^{\prime \prime}} \\
& \frac{\partial T_{k}^{\prime \prime \prime}}{\partial t}+u_{i}^{\prime \prime \prime} \frac{\partial T_{k}^{\prime \prime \prime}}{\partial x_{i}^{\prime \prime \prime}}=\frac{v}{p_{r}} \frac{\partial^{2} T_{k}^{\prime \prime \prime}}{\partial x_{i}^{\prime \prime \prime} \partial x_{i}^{\prime \prime \prime}}
\end{aligned}
$$

Where, $T, T^{\prime}, T^{\prime \prime}, T^{\prime \prime \prime}$ Temperature at point $P, P^{\prime}, P^{\prime \prime}$ and $P^{\prime \prime \prime}, u_{i}=$ Instantaneous velocity, $\rho=$ Fluid density, $c_{p}=$ Heat capacity at constant pressure, $k=$ Thermal conductivity, $x_{i}=$ Space co-ordinate, $t=$ Time, and repeated subscripts are summed from 1 to 3 .

Multiplying equation (1) by $T_{i}^{\prime} T_{j}^{\prime \prime} T_{m}^{\prime \prime \prime}$ (2) by $u_{i} T_{j}^{\prime \prime} T_{m}^{\prime \prime \prime}$ (3) by $u_{i} T_{i}^{\prime} T_{m}^{\prime \prime \prime}$ (4) by $u_{i} T_{i}^{\prime} T_{j}^{\prime \prime}$ and adding the four equations, we than taking the time averages. Time averages are denoted by $(\overline{\ldots . . .})$ We get

$$
\begin{aligned}
& \frac{\partial \overline{\left(u_{j} T_{i}^{\prime} T_{j}^{\prime \prime} T_{k}^{\prime \prime \prime}\right)}}{\partial t}+\frac{\partial \overline{\left(u_{j} u_{i} T_{i}^{\prime} T_{j}^{\prime \prime} T_{k}^{\prime \prime \prime}\right)}}{\partial x_{i}}+\frac{\partial \overline{\left(u_{i} u_{i}^{\prime} T_{i}^{\prime} T_{j}^{\prime \prime} T_{k}^{\prime \prime \prime}\right)}}{\partial x_{i}^{\prime}}+\frac{\partial \overline{\left(u_{i} u_{i}^{\prime \prime} T_{i}^{\prime} T_{j}^{\prime} T_{k}^{\prime \prime \prime}\right)}}{\partial x_{i}^{\prime \prime}}+\frac{\partial\left(u_{i} u_{j i}^{\prime \prime \prime} T_{i}^{\prime} T_{j}^{\prime \prime} T_{k}^{\prime \prime \prime}\right)}{\partial x_{i}^{\prime \prime \prime}} \\
& =-\frac{1}{\rho} \frac{\partial \overline{\left(P T_{i}^{\prime} T_{j}^{\prime \prime} T_{k}^{\prime \prime \prime}\right)}}{\partial x_{j}}+\frac{v}{P_{r}} \frac{\partial^{2}\left(\overline{\left.u_{j} T_{i}^{\prime} T_{j}^{\prime \prime} T_{k}^{\prime \prime \prime}\right)}\right.}{\partial x_{i} \partial x_{i}}+\frac{v}{p_{r}} \frac{\partial^{2}\left(\overline{u_{i} T_{i}^{\prime} T_{j}^{\prime \prime} T_{k}^{\prime \prime \prime}}\right)}{\partial x_{i}^{\prime} \partial x_{i}^{\prime}}+\frac{v}{p_{r}} \frac{\partial^{2}\left(\overline{\left.u_{i} T_{i}^{\prime} T_{j}^{\prime} T_{k}^{\prime \prime \prime}\right)}\right.}{\partial x_{i}^{\prime \prime} \partial x_{i}^{\prime \prime}}+\frac{v}{p_{r}} \frac{\partial^{2} u_{i} T_{i}^{\prime} T^{\prime \prime \prime} T_{k}^{\prime \prime \prime}}{\partial x_{i}^{\prime \prime \prime} \partial x_{i}^{\prime \prime \prime}}
\end{aligned}
$$

Using the transformation $\frac{\partial}{\partial x_{i}^{\prime \prime \prime}}=\frac{\partial}{\partial r_{i}^{\prime \prime}}, \frac{\partial}{\partial x_{i}^{\prime \prime}}=\frac{\partial}{\partial r_{i}^{\prime}}, \frac{\partial}{\partial x_{i}^{\prime}}=\frac{\partial}{\partial r_{i}}, \quad \frac{\partial}{\partial x_{i}}=-\left(\frac{\partial}{\partial r_{i}}+\frac{\partial}{\partial r_{i}^{\prime}}+\frac{\partial}{\partial r_{i}^{\prime \prime}}\right)$

in equations (5) we get,

$$
\begin{aligned}
& \frac{\partial \overline{\left(u_{j} T_{i}^{\prime} T_{j}^{\prime \prime} T_{k}^{\prime \prime \prime}\right)}}{\partial t}-\frac{\partial \overline{\left(u_{j} u_{i} T_{i}^{\prime} T_{j}^{\prime \prime} T_{k}^{\prime \prime \prime}\right)}}{\partial r_{i}}-\frac{\partial \overline{\left(u_{j} u_{i} T_{i}^{\prime} T_{j}^{\prime \prime} T_{k}^{\prime \prime \prime}\right)}}{\partial r_{i}^{\prime}}-\frac{\partial \overline{\left(u_{j} u_{i} T_{i}^{\prime} T_{j}^{\prime \prime} T_{k}^{\prime \prime \prime}\right)}}{\partial r_{i}^{\prime \prime}}+\frac{\left.\partial \overline{\left(u_{i} u_{i}^{\prime} T_{i}^{\prime} T_{j}^{\prime \prime} T_{k}^{\prime \prime \prime}\right.}\right)}{\partial r_{i}} \\
& +\frac{\partial\left(u_{i} u_{i}^{\prime \prime} T_{i}^{\prime} T_{j}^{\prime} T_{k}^{\prime \prime \prime}\right)}{\partial r_{i}^{\prime}}+\frac{\partial\left(u_{i} u_{j}^{\prime \prime \prime} T_{i}^{\prime} T_{j}^{\prime \prime} T_{k}^{\prime \prime \prime}\right)}{\partial r_{i}^{\prime \prime}}=\frac{1}{\rho}\left[\frac{\partial \overline{\left(P T_{i}^{\prime} T_{j}^{\prime \prime} T_{k}^{\prime \prime \prime}\right)}}{\partial r_{j}}+\frac{\partial \overline{\left(P T_{i}^{\prime} T_{j}^{\prime \prime} T_{k}^{\prime \prime \prime}\right)}}{\partial r_{j}^{\prime}}+\frac{\partial \overline{\left(P T_{i}^{\prime} T_{j}^{\prime \prime} T_{k}^{\prime \prime \prime}\right)}}{\partial r_{j}^{\prime \prime}}\right] \\
& +\left(1+P_{r}\right) \frac{\partial^{2}\left(\overline{\left.u_{i} T_{i}^{\prime} T_{j}^{\prime} T_{k}^{\prime \prime \prime}\right)}\right.}{\partial r_{i}^{\prime} \partial r_{i}^{\prime}}+\left(1+P_{r}\right) \frac{\partial^{2}\left(u_{i} T_{i}^{\prime} T^{\prime \prime} T_{k}^{\prime \prime \prime}\right)}{\partial r_{i}^{\prime \prime} \partial r_{i}^{\prime \prime}}+2 P_{r} \frac{\partial^{2}\left(u_{i} T_{i}^{\prime} T_{j}^{\prime} T_{k}^{\prime \prime \prime}\right)}{\partial r_{i} \partial r_{i}^{\prime}}+2 P_{r} \frac{\partial^{2}\left(u_{i} T_{i}^{\prime} T_{j}^{\prime} T_{k}^{\prime \prime \prime}\right)}{\partial r_{i}^{\prime} \partial r_{i}^{\prime \prime}}
\end{aligned}
$$


In order to convert the equation (6) into spectral form, by using the nine dimensional Fourier transforms we get

$$
\begin{aligned}
& \frac{\partial}{\partial t}\left(\overline{\phi_{l} \gamma^{\prime} \gamma^{\prime \prime} \gamma^{\prime \prime \prime}}\right)+\frac{v}{P_{r}}\left[\left(1+P_{r}\right)\left(K^{2}+\left(1+P_{r}\right) K^{\prime 2}+\left(1+P_{r}\right) K^{\prime \prime 2}+2 P_{r} K K^{\prime}+2 P_{r} K K^{\prime \prime}+2 P_{r} K K^{\prime \prime \prime}\right] \overline{\left(\phi_{l} \gamma^{\prime} \gamma^{\prime \prime} \gamma^{\prime \prime \prime}\right.}\right)= \\
& i\left(K_{k}+K_{k}^{\prime}+K^{\prime \prime}\right)\left(\overline{\phi_{l} \phi_{k} \gamma_{i}^{\prime} \gamma_{j}^{\prime \prime} \gamma_{m}^{\prime \prime \prime}}\right)-i\left(K_{k}+K_{k}^{\prime}+K^{\prime \prime}\right)\left(\overline{\phi_{l} \phi_{k}^{\prime} \gamma_{i}^{\prime} \gamma_{j}^{\prime \prime} \gamma_{m}^{\prime \prime \prime}}\right) \\
& +i\left(K_{k}+K_{k}^{\prime}+K^{\prime \prime}\right)\left(\overline{\gamma_{l} \gamma_{k}^{\prime} \gamma_{i}^{\prime} \gamma_{j}^{\prime \prime} \gamma_{m}^{\prime \prime \prime}}\right)
\end{aligned}
$$

The tensor equation (7) can be converted to the scalar equation by contraction of the indices $\mathrm{i}$ and $\mathrm{j}$;

$$
\begin{aligned}
& \frac{\partial}{\partial t}\left(\overline{\phi_{l} \gamma^{\prime} \gamma^{\prime \prime} \gamma^{\prime \prime \prime}}\right)+\frac{v}{P_{r}}\left[\left(1+P_{r}\right)\left(K^{2}+\left(1+P_{r}\right) K^{\prime 2}+\left(1+P_{r}\right) K^{\prime \prime 2}+2 P_{r} K K^{\prime}+2 P_{r} K K^{\prime \prime}+2 P_{r} K K^{\prime \prime \prime}\right]\left(\overline{\phi_{l} \gamma^{\prime} \gamma^{\prime \prime} \gamma^{\prime \prime \prime}}\right)=\right. \\
& i\left(K_{k}+K_{k}^{\prime}+K^{\prime \prime}\right)\left(\overline{\phi_{l} \phi_{k} \gamma_{i}^{\prime} \gamma_{i}^{\prime \prime} \gamma_{m}^{\prime \prime \prime}}\right)-i\left(K_{k}+K_{k}^{\prime}+K^{\prime \prime}\right)\left(\overline{\gamma_{l} \gamma_{k} \gamma_{i}^{\prime} \gamma_{i}^{\prime \prime} \gamma_{m}^{\prime \prime \prime}}\right)-i\left(K_{k}+K_{k}^{\prime}+K^{\prime \prime}\right)\left(\overline{\phi_{l} \phi_{k}^{\prime} \gamma_{i}^{\prime} \gamma_{i}^{\prime \prime} \gamma_{m}^{\prime \prime \prime}}\right) \\
& +i\left(K_{k}+K_{k}^{\prime}+K^{\prime \prime}\right)\left(\overline{\gamma_{l} \gamma_{k}^{\prime} \gamma_{i}^{\prime} \gamma_{i}^{\prime \prime} \gamma_{m}^{\prime \prime \prime}}\right)+i \frac{1}{\rho}\left(K_{k}+K_{k}^{\prime}+K^{\prime \prime}\right)\left(\overline{P \gamma_{i}^{\prime} \gamma_{i}^{\prime \prime} \gamma_{m}^{\prime \prime \prime}}\right)
\end{aligned}
$$

If we take the derivative with respect to $x_{i}$ of the momentum equation (1) at $\mathrm{p}$, we have

$$
\frac{\partial^{2} u_{j} u_{i}}{\partial x_{i} \partial x_{i}}=-\frac{1}{\rho} \frac{\partial^{2} P}{\partial x_{i} \partial x_{j}}
$$

Multiplying equation (9) by $T_{i}^{\prime} T_{j}^{\prime \prime} T_{m}^{\prime \prime \prime}$; taking time averages

$$
\frac{\partial^{2} \overline{\left(u_{j} u_{i} T_{i} T_{j}^{\prime \prime} T_{m}^{\prime \prime \prime}\right)}}{\partial x_{i} \partial x_{i}}=-\frac{1}{\rho} \frac{\partial^{2} \overline{\left(P T_{i} T_{j}^{\prime \prime} T_{m}^{\prime \prime \prime}\right)}}{\partial x_{i} \partial x_{j}}
$$

Writting the equation (10) in terms of the independent variables $\vec{r}, \overrightarrow{r^{\prime}}, \overrightarrow{r^{\prime \prime}}$ we have,

$$
\begin{aligned}
& \frac{\partial^{2}}{\partial r_{l} \partial r_{l}}+\frac{\partial^{2}}{\partial r_{l}^{\prime} \partial r_{l}^{\prime}}+\frac{\partial^{2}}{\partial r_{l}^{\prime \prime} \partial r_{l}^{\prime \prime}}+\frac{\partial^{2}}{\partial r_{l} \partial r_{l}^{\prime}}+\frac{\partial^{2}}{\partial r_{l}^{\prime} \partial r_{l}^{\prime \prime}}+\frac{\partial^{2}}{\partial r_{l} \partial r_{l}^{\prime \prime}} \frac{\left(u_{j} u_{i} T_{i}^{\prime} T_{j}^{\prime \prime} T_{m}^{\prime \prime \prime}\right)}{=}= \\
& -\frac{1}{\rho}\left[\frac{\partial^{2}}{\partial r_{l} \partial r_{k}}+\frac{\partial^{2}}{\partial r_{l} \partial r_{k}^{\prime}}+\frac{\partial^{2}}{\partial r_{l}^{\prime} \partial r_{k}^{\prime}}+\frac{\partial^{2}}{\partial r_{l}^{\prime} \partial r_{k}^{\prime \prime}}+\frac{\partial^{2}}{\partial r_{l}^{\prime} \partial r_{k}^{\prime \prime}}+\frac{\partial^{2}}{\partial r_{l} \partial r_{l}^{\prime \prime}}+\frac{\partial^{2}}{\partial r_{l}^{\prime \prime} \partial r_{k}^{\prime}}+\frac{\partial^{2}}{\partial r_{l}^{\prime \prime} \partial r_{k}^{\prime \prime}} \overline{\left(P T_{i} T_{j}^{\prime \prime} T_{m}^{\prime \prime \prime}\right)}\right.
\end{aligned}
$$

and

$$
-\frac{1}{\rho} \overline{\left(P \gamma_{i}^{\prime} \gamma_{j}^{\prime \prime} \gamma_{k}^{\prime \prime \prime}\right)}=\frac{K_{l} K_{k}+K_{l} K_{k}^{\prime}+K_{l}^{\prime} K_{k}+K_{l}^{\prime} K_{k}^{\prime}+K_{l}^{\prime \prime} K_{k}+K_{l} K_{k}^{\prime \prime}+K_{l}^{\prime} K_{k}^{\prime \prime}+K_{l}^{\prime \prime} K_{k}^{\prime}+K_{l}^{\prime \prime} K_{k}^{\prime \prime}}{K_{l} K_{l}+K_{l}^{\prime} K_{l}^{\prime}+K_{l}^{\prime \prime} K_{l}^{\prime \prime}+2 K_{l} K_{l}^{\prime}+2 K_{l}^{\prime \prime} K_{l}+2 K_{l}^{\prime} K_{l}^{\prime \prime} \gamma_{i}^{\prime \prime} \gamma_{m}^{\prime \prime \prime}}
$$

Equation (12) can be used to eliminate $\left(\overline{-\frac{1}{\rho} P \gamma_{i}^{\prime} \gamma_{j}^{\prime \prime} \gamma_{m}^{\prime \prime \prime}}\right)$ from equation (8) and (10) if we take contraction of the indices $\mathrm{i}$ and $\mathrm{j}$ in equation (12).

Equations (11) and (12) are the spectral equation corresponding to the four -point correlation equation. The spectral equations corresponding to the three-point correlation equations by contraction of the indices $\mathrm{i}$ and $\mathrm{m}$ are

$$
\begin{aligned}
& \frac{\partial}{\partial t}\left(\overline{\phi_{i} \beta_{i}^{\prime} \beta_{i}^{\prime \prime}}\right)+\frac{v}{P_{r}}\left[\left(1+P_{r}\right)\left(K^{2}+K^{\prime 2}+2 P_{r} K K^{\prime}\right]\left(\overline{\phi_{i} \beta_{i}^{\prime} \beta_{i}^{\prime \prime}}\right)=i\left(K_{k}+K_{k}^{\prime}\right)\left(\overline{\phi_{i} \phi_{k} \beta_{i}^{\prime} \beta_{i}^{\prime \prime}}\right)\right. \\
& -i\left(K_{k}+K_{k}^{\prime}\right)\left(\overline{\beta_{i} \beta_{k} \beta_{i}^{\prime} \beta_{i}^{\prime \prime}}\right)-i\left(K_{k}+K_{k}^{\prime}\right)\left(\overline{\phi_{i} \phi_{k}^{\prime} \beta_{i}^{\prime} \beta_{i}^{\prime \prime}}\right)+i\left(K_{k}+K_{k}^{\prime}\right)\left(\overline{\phi_{i}^{\prime} \phi_{k} \beta_{i}^{\prime} \beta_{i}^{\prime \prime}}\right)+i\left(K_{k}+K_{k}^{\prime}\right)\left(\overline{\gamma \beta_{i}^{\prime} \beta_{i}^{\prime \prime}}\right)
\end{aligned}
$$

And, $\quad-\quad-\frac{1}{\rho}\left(\overline{\gamma \beta_{i}^{\prime} \beta_{i}^{\prime \prime}}\right)=\frac{K_{i} K_{k}+K_{i} K_{k}^{\prime}+K_{i}^{\prime} K_{k}+K_{i}^{\prime} K_{k}^{\prime}}{K_{l}^{2}+K_{l}^{\prime 2}+2 K_{i} K_{i}^{\prime}}\left(\overline{\phi_{i}^{\prime} \phi_{k}^{\prime} \beta_{i}^{\prime \prime}}\right)$

using six dimensional Fourier transforms we get from (13)The spectral equations corresponding to the twopoint correlation equations by contraction of the indices $\mathrm{i}$ and $\mathrm{j}$ are

$$
\frac{\partial}{\partial t}\left(\overline{\phi_{i} \beta_{i}^{\prime \prime}}\right)+\frac{2 v}{P_{r}} K^{2}\left(\overline{\phi_{i} \beta_{i}^{\prime}}\right)=2 i K_{k}\left[\left(\overline{\phi_{i} \phi_{k}^{\prime} \beta_{i}^{\prime \prime}}\right)-\left(\overline{\phi_{i}^{\prime} \phi_{k} \beta_{i}^{\prime \prime}}\right)\right.
$$

\section{Solution Neglecting Quintuple Correlations-}

As it stands the set of linear equations (8),(13),(15) are indeterminate as it contains more unknowns than equations in equation (15).For this to find the solution we can neglecting all the terms on the right side of equation (15), the equation can be integrated between $t_{1}$ and to give 


$$
\left\langle\phi_{l} \gamma_{i}^{\prime} \gamma_{j}^{\prime \prime} \gamma_{m}^{\prime \prime \prime}\right\rangle=\left\langle\phi_{l} \gamma_{i}^{\prime} \gamma_{j}^{\prime \prime} \gamma_{m}^{\prime \prime \prime}\right\rangle_{1} \exp \left[\begin{array}{l}
\left\{\frac{-v}{p_{r}}\left(1+p_{r}\right)\left(k^{2}+k^{\prime 2}+k^{\prime \prime 2}+2 k k^{\prime}+2 k^{\prime} k^{\prime \prime}+2 k k^{\prime}\right)\right\} \\
\left(t-t_{1}\right)
\end{array}\right]
$$

where $\left\langle\phi_{l} \gamma_{i}^{\prime} \gamma_{j}^{\prime \prime} \gamma_{m}^{\prime \prime \prime}\right\rangle_{1}$ is the value of $\left\langle\phi_{l} \gamma_{i}^{\prime} \gamma_{j}^{\prime \prime} \gamma_{m}^{\prime \prime \prime}\right\rangle$ at $\mathrm{t}=t_{1}$ that is stationary value for small values of $\mathrm{k}, k^{\prime}$ and $k^{\prime \prime}$ when the quintuple correlations are negligible.

Substituting of equation (12), (14), (16) in equation (13) (R. G. Deissler, [3]

We get

$$
\frac{\partial}{\partial t}\left(k_{k} \overline{\phi_{i} \beta_{i}^{\prime} \beta_{i}^{\prime \prime}}\right)+\frac{v}{P_{r}}\left[\left(1+P_{r}\right)\left(K^{2}+K^{\prime 2}+2 P_{r} K K^{\prime}\right]\left(k_{k} \overline{\phi_{i} \beta_{i}^{\prime} \beta_{i}^{\prime \prime}}\right)=\right.
$$

$[a]_{1} \int_{-\infty}^{\infty} \exp \left[-\frac{v}{P_{r}}\left(t-t_{1}\right)\left[\left\{\left(1+P_{r}\right)\left(k^{2}+k^{\prime 2}+k^{\prime \prime 2}\right)+2 p_{r}\left(k k^{\prime}+k^{\prime} k^{\prime \prime}+k^{\prime \prime} k\right)\right\} d k^{\prime \prime}\right]+\right.$

$[b]_{1} \int_{-\infty}^{\infty} \exp \left[-\frac{v}{P_{r}}\left(t-t_{1}\right)\left[\left\{\left(1+P_{r}\right)\left(k^{2}+k^{\prime 2}+k^{\prime \prime 2}\right)+2 p_{r} k k^{\prime}\right\} d k^{\prime \prime}\right]+\right.$

$[c]_{1} \int_{-\infty}^{\infty} \exp \left[-\frac{v}{P_{r}}\left(t-t_{1}\right)\left[\left\{\left(1+P_{r}\right)\left(k^{2}+k^{\prime 2}+k^{\prime \prime 2}\right)+2 p_{r} k k^{\prime}\right\} d k^{\prime \prime}\right]\right.$

At $t_{1}$, functions $\gamma$ 's have been assumed independent that assumption is not, made for other times. This is one of several assumptions made concerning the initial conditions, although continuity equation satisfied the conditions. The complete specification of initial condition is difficult; the assumptions for the initial conditions made here, in are partially on the basis of simplicity. Substituting $d k^{\prime \prime}=d k_{1}^{\prime \prime} d k_{2}^{\prime \prime} d k_{3}^{\prime \prime}$ integrating equation (17) with respect to $k_{1}^{\prime \prime}, k_{2}^{\prime \prime}$ and $k_{2}^{\prime \prime \prime}$,

$$
\begin{aligned}
& \text { we get, } \quad \frac{\partial}{\partial t}\left(k_{k} \overline{\phi_{i} \beta_{i}^{\prime} \beta_{i}^{\prime \prime}}\right)+\frac{v}{P_{r}}\left[\left(1+P_{r}\right)\left(K^{2}+K^{\prime 2}+2 P_{r} K K^{\prime}\right]\left(k_{k} \overline{\phi_{i} \beta_{i}^{\prime} \beta_{i}^{\prime \prime}}\right)=\left(\frac{\pi p_{r}}{v\left(t-t_{1}\right)\left(1+p_{r}\right)}\right)^{\frac{3}{2}}[\mathrm{a}]_{1}\right. \\
& \exp \left[-\frac{\nu\left(t-t_{1}\right)\left[\left(1+P_{r}\right)\right.}{P_{r}}\left\{\frac{\left(1+2 P_{r}\right)\left(k^{2}+k^{\prime 2}\right)}{\left(1+P_{r}\right)^{2}}+\frac{2 P_{r} k k^{\prime}}{\left(1+P_{r}\right)^{2}}\right\}\right]+\left(\frac{\pi p_{r}}{v\left(t-t_{1}\right)\left(1+p_{r}\right)}\right)^{\frac{3}{2}}[\mathrm{~b}]_{1} \\
& \exp \left[-\frac{\nu\left(t-t_{1}\right)\left[\left(1+P_{r}\right)\right.}{P_{r}}\left\{\frac{\left(1+2 P_{r}\right) k^{2}}{\left(1+P_{r}\right)^{2}}+\frac{2 P_{r} k k^{\prime}}{\left(1+P_{r}\right)}+k^{\prime 2}\right\}\right]+\left(\frac{\pi p_{r}}{v\left(t-t_{1}\right)\left(1+p_{r}\right)}\right)^{\frac{3}{2}}[\mathrm{c}]_{1} \\
& \exp \left[-\frac{v\left(t-t_{1}\right)\left[\left(1+P_{r}\right)\right.}{P_{r}}\left\{k^{2}+\frac{\left(1+2 P_{r}\right)\left(k^{\prime 2}\right)}{\left(1+P_{r}\right)^{2}}+\frac{2 P_{r} k k^{\prime}}{\left(1+P_{r}\right)}\right\}\right]
\end{aligned}
$$

Integration of equation (18) with respect to time, and in order to simplify calculations, we will assume that $[a]_{1}=0$; That is we assume that a function sufficiently general to represent the initial conditions can obtained by considering only the terms involving $[b]_{1}$ and $[c]_{1}$ The substituting of equation (15) in equation (13) and setting $T=2 \pi k^{2} \varphi_{i} \varphi_{i}^{\prime}$, result in

$$
\frac{\partial T}{\partial t}+\frac{2 v k^{2}}{p_{r}} T=E
$$

where,

$$
\begin{aligned}
& \mathrm{E}=k^{2} \int_{-\infty}^{\infty} 2 \pi . i\left[\left\langle k_{k} \phi_{l} \beta_{i}^{\prime} \beta_{i}^{\prime \prime}\left(\hat{k}, \hat{k}^{\prime}\right)\right\rangle-\left\langle k_{k} \phi_{l} \beta_{i}^{\prime} \beta_{i}^{\prime \prime}\left(-\hat{k},-\hat{k}^{\prime}\right)\right\rangle\right]_{0} . \\
& \exp \left[-\frac{v}{P_{r}}\left(t-t_{1}\right)\left\{\left(1+P_{r}\right)\left(k^{2}+k^{\prime 2}\right)+2 p_{r} k k^{\prime}\right\} d k^{\prime}\right]+k^{2} \int_{-\infty}^{\infty} \frac{2 p_{r} \cdot \pi^{\frac{5}{2}}}{v} i\left[b\left(\hat{k} \cdot \hat{k}^{\prime}\right)-b\left(-\hat{k} \cdot-\hat{k}^{\prime}\right)\right] \cdot\{-
\end{aligned}
$$




$$
\begin{aligned}
& \omega^{-1} \exp \left[\left(-\omega^{2}\right)\left\{\frac{\left(1+2 p_{r}\right)\left(k^{2}\right)}{\left(1+p_{r}\right)^{2}}+\frac{2 p_{r} k k^{\prime}}{\left(1+p_{r}\right)}+k^{\prime 2}\right\}\right]+\operatorname{kexp}\left[\left(-\omega^{2}\right)\left(\left(1+p_{r}\right)\left(k^{2}+k^{\prime 2}\right)+2 p_{r} k k^{\prime}\right)\right. \\
& ] \times \int_{0}^{\omega k / 2} \exp \left(x^{2}\right) d x\right\} d k+k^{2} \int_{-\infty}^{\infty} \frac{2 p_{r} \cdot \pi^{\frac{5}{2}}}{v} i\left[c\left(\hat{k} \cdot \hat{k}^{\prime}\right)-c\left(-\hat{k} \cdot-\hat{k}^{\prime}\right)\right] . \\
& \omega^{-1} \exp \left[\left(-\omega^{2}\right)\left\{k^{2}+\frac{\left(1+2 p_{r}\right)\left(k^{\prime 2}\right)}{\left(1+p_{r}\right)^{2}}+\frac{2 p_{r} k k^{\prime}}{\left(1+p_{r}\right)}\right\}\right] k^{\prime}(\operatorname{expk}) \times \exp \left[\left(-\omega^{2}\right)\left(\left(1+p_{r}\right)\left(k^{2}+k^{\prime 2}\right)+2 p_{r} k k^{\prime}\right)\right] \\
& \left.\int_{0}^{\omega k^{\prime} / 2} \exp \left(x^{2}\right) d x\right\} d k^{\prime}
\end{aligned}
$$

Where $\mathrm{T}$ is the temperature fluctuations spectrum function, which represent contributions from various wave numbers (or eddy sizes) to the energy and $\mathrm{E}$ is the energy transfer function, which is responsible for the transfer of energy between wave numbers. In order to make further calculations, an assumption must be made for the forms of the bracketed quantities with the subscripts 0 and 1 in equation (20) which depends on the initial conditions.

$$
(2 \pi)^{2}\left[\left\langle k_{k} \phi_{l} \beta_{i}^{\prime} \beta_{i}^{\prime \prime}\left(\hat{k}, \hat{k}^{\prime}\right)\right\rangle-\left\langle k_{k} \phi_{l} \beta_{i}^{\prime} \beta_{i}^{\prime \prime}\left(-\hat{k},-\hat{k}^{\prime}\right)\right\rangle\right]_{0}=-\xi_{0}\left(k^{2} k^{\prime 4}-k^{4} k^{\prime 2}\right)
$$

Where, $\xi_{0}$ is a constant depending on the initial conditions .

For the other bracketed quantities in equation (20), We get,

$$
\frac{4 p_{r} \cdot \pi^{\frac{7}{2}}}{v} i\left[b\left(\hat{k} \cdot \hat{k}^{\prime}\right)-b\left(-\hat{k} \cdot-\hat{k}^{\prime}\right)\right]=\frac{4 p_{r} \cdot \pi^{\frac{7}{2}}}{v} i\left[c\left(\hat{k} \cdot \hat{k}^{\prime}\right)-c\left(-\hat{k} \cdot-\hat{k}^{\prime}\right)\right]=-2 \xi_{1}\left(k^{4} k^{\prime 6}-k^{6} k^{\prime 4}\right)
$$

Remembering that $d \hat{k}^{\prime}=-2 \pi \cdot \hat{k}^{\prime 2} d(\cos \theta)$ and $k k^{\prime}=k k^{\prime} \cos \theta, \theta$ is the angle between $\hat{k}$ and $\hat{k}^{\prime}$ and carrying out the integration with respect to $\theta$, we get,

$$
\begin{aligned}
& \mathrm{E}=-\int_{0}^{\infty}\left[\frac { \xi _ { 0 } ( k ^ { 2 } k ^ { \prime 4 } - k ^ { 4 } k ^ { \prime 2 } ) k k ^ { \prime } } { v ( t - t _ { 0 } ) } \left\{\exp \left[-\frac{v}{P_{r}}\left(t-t_{0}\right)\left\{\left(1+P_{r}\right)\left(k^{2}+k^{\prime 2}\right)-2 p_{r} k k^{\prime}\right\}\right]-\right.\right. \\
& \left.\exp \left[-\frac{v}{P_{r}}\left(t-t_{0}\right)\left\{\left(1+P_{r}\right)\left(k^{2}+k^{\prime 2}\right)+2 p_{r} k k^{\prime}\right\}\right]\right\}+\frac{\xi_{1}\left(k^{4} k^{\prime 6}-k^{6} k^{\prime 4}\right) k k^{\prime}}{v\left(t-t_{0}\right)} . \\
& -\left(-\omega^{-1}\right) \omega^{-1} \exp \left[\left(-\omega^{2}\right)\left\{\frac{\left(1+2 p_{r}\right)\left(k^{2}\right)}{\left(1+p_{r}\right)^{2}}+\frac{2 p_{r} k k^{\prime}}{\left(1+p_{r}\right)}+k^{\prime 2}\right\}\right] . \\
& +\left(-\omega^{-1}\right) \omega^{-1} \exp \left[\left(-\omega^{2}\right)\left\{k^{2}-\frac{\left(1+2 p_{r}\right)\left(k^{\prime 2}\right)}{\left(1+p_{r}\right)^{2}}+\frac{2 p_{r} k k^{\prime}}{\left(1+p_{r}\right)}+k^{\prime 2}\right\}\right]- \\
& \left(-\omega^{-1}\right) \omega^{-1} \exp \left[\left(-\omega^{2}\right)\left\{\frac{\left(1+2 p_{r}\right)\left(k^{2}\right)}{\left(1+p_{r}\right)^{2}}+\frac{2 p_{r} k k^{\prime}}{\left(1+p_{r}\right)}+k^{2}\right\}\right]+\mathrm{k} \cdot \exp \left[\left(-\omega^{2}\right)\left(\left(1+p_{r}\right)\left(k^{2}+k^{\prime 2}\right)-2 p_{r} k k^{\prime}\right)\right]- \\
& \left.\mathrm{k} \cdot \exp \left[\left(-\omega^{2}\right)\left(\left(1+p_{r}\right)\left(k^{2}+k^{\prime 2}\right)+2 p_{r} k k^{\prime}\right)\right]\right\} \int_{0}^{\frac{\omega k}{2}} \exp \left(x^{2}\right) d x+ \\
& \left\{k^{\prime} \exp \left[-\omega^{2}\left(\left(1+p_{r}\right)\left(k^{2}+k^{\prime 2}\right)-2 p_{r} k k^{\prime}\right)\right]-\right.
\end{aligned}
$$


$\left.\left.k^{\prime} \exp \left[-\omega^{2}\left(\left(1+p_{r}\right)\left(k^{2}+k^{\prime 2}\right)+2 p_{r} k k^{\prime}\right)\right]\right\} \int_{0}^{\frac{\omega k}{2}} \exp \left(x^{2}\right) d x\right] d k^{\prime}$

Where, $\quad \omega=\left(\frac{v\left(t-t_{1}\right)\left[\left(1+P_{r}\right)\right.}{P_{r}}\right)^{\frac{1}{2}}$

Integrating, equation (23) with respect to $k^{\prime}$.

We have, $E=E_{\beta}+E_{\gamma}$

Where,

$$
\begin{aligned}
& E_{\beta}=\frac{\pi^{\frac{1}{2}} \xi_{0} p_{r}}{v^{\frac{3}{2}}\left(t-t_{0}\right)^{\frac{3}{2}}\left(1+p_{r}\right)^{\frac{5}{2}}} \exp \left\{\frac{v\left(t-t_{0}\right)\left(1+p_{r}\right) k^{2}}{p_{r}\left(1+p_{r}\right)}\right\}\left[\frac{15 p_{r} k^{4}}{4 v^{2}\left(t-t_{0}\right)^{2}\left(1+p_{r}\right)}+\right. \\
& \left.\left\{\frac{5 p_{r}{ }^{2}}{\left(1+p_{r}\right)^{2} v\left(t-t_{0}\right)}-\frac{3}{2 v\left(t-t_{0}\right)}\right\} k^{6}+\frac{p_{r}}{\left(1+p_{r}\right)}\left\{\frac{p_{r}{ }^{2}}{\left(1+p_{r}\right)^{2}}\right\} k^{8}\right]
\end{aligned}
$$

And

$$
\begin{aligned}
& E_{\gamma}=\frac{\pi^{\frac{1}{2}} \xi_{1} p_{r}{ }^{5}}{8 v^{2}\left(t-t_{1}\right)^{\frac{3}{2}}\left(1+p_{r}\right)^{5}} \exp \left\{\frac{-v\left(t-t_{1}\right)\left(1+2 p_{r}-p_{r}{ }^{2}\right)}{p_{r}\left(1+p_{r}\right)}\right\} k^{2}\left[\frac{90 p_{r} k^{6}}{v^{4}\left(t-t_{1}\right)^{4}\left(1+p_{r}\right)}+\right. \\
& 3\left\{\frac{4 p_{r}}{\left(1+p_{r}\right) v^{2}\left(t-t_{1}\right)^{2}}+\frac{2 p_{r}{ }^{2}}{\left(1+p_{r}\right)^{2} v^{3}\left(t-t_{1}\right)^{3}}-\frac{1}{v^{3}\left(t-t_{1}\right)^{3}}\right\} k^{8}+ \\
& \left.\left\{\frac{64 p_{r}{ }^{2}}{\left(1+p_{r}\right)^{2} v\left(t-t_{1}\right)}+\frac{10 p_{r}{ }^{3}}{\left(1+p_{r}\right)^{3} v^{2}\left(t-t_{1}\right)^{2}}-\frac{40}{v\left(t-t_{1}\right)}\right\} k^{10}+8\left\{\frac{p_{r}{ }^{2}}{\left(1+p_{r}\right)^{2}}+\frac{p_{r}}{\left(1+p_{r}\right)}\right\} k^{12}\right]+ \\
& \frac{\pi^{\frac{1}{2}} \xi_{1} p_{r}{ }^{5}\left(1+p_{r}\right)^{4}}{8 v^{2}\left(t-t_{1}\right)^{2}\left(1+2 p_{r}\right)^{\frac{9}{2}}} \exp \left\{\frac{-v\left(t-t_{1}\right)\left(1+p_{r}\right)\left(1+2 p_{r}-p_{r}{ }^{2}\right)}{p_{r}\left(1+p_{r}\right)}\right\} k^{2} \cdot\left[\frac{90 p_{r}\left(1+p_{r}\right) k^{6}}{v^{4}\left(t-t_{1}\right)^{4}\left(1+2 p_{r}\right)}+\right. \\
& \left\{\frac{120 p_{r}\left(1+p_{r}\right)}{v^{2}\left(t-t_{1}\right)^{2}\left(1+2 p_{r}\right)}+\frac{2 p_{r}{ }^{2}\left(1+p_{r}\right)^{2}}{\left(1+p_{r}\right)^{2} v^{3}\left(t-t_{1}\right)^{3}}-\frac{1}{v^{3}\left(t-t_{1}\right)^{3}}\right\} k^{8}+ \\
& \left.\left\{\frac{64 p_{r}{ }^{2}\left(1+p_{r}\right)^{2}}{\left(1+2 p_{r}\right)^{2} v\left(t-t_{1}\right)}+\frac{10 p_{r}{ }^{3}\left(1+p_{r}\right)^{3}}{\left(1+2 p_{r}\right)^{3} v^{2}\left(t-t_{1}\right)^{2}}-\frac{40}{v\left(t-t_{1}\right)}\right\} k^{10}+\left\{\frac{8 p_{r}{ }^{3}\left(1+p_{r}\right)^{3}}{\left(1+2 p_{r}\right)^{2}}-\frac{p_{r}\left(1+p_{r}\right)}{\left(1+2 p_{r}\right)}\right\} k^{12}\right]+ \\
& \left\{\frac{64 p_{r}{ }^{2}}{v\left(t-t_{1}\right)}+\frac{60 p_{r}{ }^{3}}{v^{2}\left(t-t_{1}\right)^{2}\left(1+p_{r}\right)^{2}}-\frac{40\left(1+p_{r}\right)^{2}}{v\left(t-t_{1}\right)}\right\} k^{11}+\left\{p_{r}{ }^{2}-p_{r}\left(1+p_{r}\right)^{2}\right\} \int_{0}^{2} \exp \left(y^{2}\right) d y \\
& \text { where, } \omega_{1}=\left(\frac{v\left(t-t_{1}\right)\left[\left(1+P_{r}\right)\right.}{P_{r}}\right)^{\frac{1}{2}} k
\end{aligned}
$$

The integral expression in equation (24), the quantity $E_{\beta}$ represents the transfer function arising owing to consideration of Temperature fluctuation field at three point correlation equation; $E_{\gamma}$ arises from consideration of the four -point correlation equation. Integration of equation (24) over all wave numbers shows that $\int_{0}^{\infty} E \cdot d \vec{k}=0$

Indicating that the expression for E satisfies the conditions of continuity and homogeneity, physically, it was to be expected, Since $E$ is a measure of transfer of energy and the numbers must be zero. From (19), we get,

$$
T=\exp \left[-\frac{2 v k^{2}\left(t-t_{0}\right)}{p_{r}}\right] \int E \exp \left[-\frac{2 v k^{2}\left(t-t_{0}\right)}{p_{r}}\right] d t+J(k) \exp \left[-\frac{2 v k^{2}\left(t-t_{0}\right)}{p_{r}}\right]
$$


$=J(k) \exp \left[-\frac{2 v k^{2}\left(t-t_{0}\right)}{p_{r}}\right]+\exp \left[-\frac{2 v k^{2}\left(t-t_{0}\right)}{p_{r}}\right] \int\left(E_{\beta}+E_{\gamma}\right) \exp \left[-\frac{2 v k^{2}\left(t-t_{0}\right)}{p_{r}}\right] d t$

Where, $J(k)=\frac{N_{0} k^{2}}{\pi}$ is a constant of integration and can be obtained as by Corrisin S. [3]

Where, $E=E_{\beta}+E_{\gamma}$

After integration, equation (27) becomes

$T=J(k) \exp \left[-\frac{2 k^{2}\left(t-t_{0}\right)}{p_{r}}\right]+T_{\beta}+T_{\gamma}=T_{\alpha}+T_{\beta}+T_{\gamma}$

Where,

$$
\begin{aligned}
& T_{\beta}=\frac{\pi^{\frac{1}{2}} \xi_{0} p_{r}^{\frac{5}{2}}}{8 v^{\frac{3}{2}}\left(1+p_{r}\right)^{\frac{7}{2}}} \exp \left\{-\frac{v\left(t-t_{0}\right)\left(1+2 p_{r}\right) k^{2}}{p_{r}\left(1+p_{r}\right)}\right\}+\left[\frac{3 p_{r} k^{4}}{\left.2 v^{2}\left(t-t_{0}\right)^{\frac{5}{2}}\right)}+\right. \\
& \left.\left\{\frac{5 p_{r}{ }^{2}-6 p_{r}}{3 v\left(1+p_{r}\right)\left(t-t_{0}\right)^{\frac{3}{2}}}\right\} k^{6}-\left\{\frac{3 p_{r}{ }^{2}-2 p_{r}+3}{3\left(1+p_{r}{ }^{2}\right)\left(t-t_{0}\right)^{\frac{1}{2}}}\right\} k^{8}+\left\{\frac{8 v^{\frac{1}{2}}\left(3 p_{r}{ }^{2}-2 p_{r}+3\right.}{3\left(1+p_{r}\right)^{\frac{5}{2}} p_{r}{ }^{\frac{1}{2}}}\right\} k^{9} F(\omega)\right] \\
& \text { Where, } \mathrm{F}(\omega)=\exp \left(-\omega^{2}\right) \int_{0}^{\omega} \exp \left(x^{2}\right) d x, \omega=\left[\frac{v\left(t-t_{0}\right)}{p_{M}\left(1+p_{M}\right)}\right]^{\frac{1}{2}} k \\
& T_{\gamma}=\frac{\pi^{\frac{1}{2}} \xi_{1} p_{r}{ }^{5}}{8 v^{2}\left(1+p_{r}\right)^{5}} \exp \left\{\frac{-v\left(t-t_{1}\right)\left(1+2 p_{r}-p_{r}{ }^{2}\right)}{p_{r}\left(1+p_{r}\right)}\right\} k^{2}\left[\frac{18 p_{r} k^{6}}{v^{4}\left(t-t_{1}\right)^{5}\left(1+p_{r}\right)}+\right. \\
& \left\{\frac{15-6 p_{r}+21 p_{r}{ }^{2}}{4\left(1+p_{r}\right)^{2} v^{3}\left(t-t_{1}\right)^{4}}+\frac{4 p_{r}}{\left(1+p_{r}\right) v^{2}\left(t-t_{1}\right)^{3}}-\frac{1}{v^{3}\left(t-t_{1}\right)^{3}}\right\} k^{8}+ \\
& \left\{\frac{15-6 p_{r}+36 p_{r}{ }^{2}-6 p_{r}{ }^{4}}{12\left(1+p_{r}\right)^{3} v^{2}\left(t-t_{1}\right)^{3}}+\frac{14 p_{r}{ }^{2}-404 p_{r}-18}{\left(1+p_{r}\right)^{2} v\left(t-t_{1}\right)}\right\} k^{10} \cdot \exp \left(-\omega_{1}\right) \int E i\left(\omega_{1}\right) d t+ \\
& \frac{\pi^{\frac{1}{2}} \xi_{1} p_{r}^{5}\left(1+p_{r}\right)^{4}}{8 v^{2}\left(1+2 p_{r}\right)^{\frac{9}{2}}} \exp \left\{\frac{-v\left(t-t_{1}\right)\left(1+2 p_{r}-p_{r}{ }^{2}\right)}{p_{r}\left(1+p_{r}\right)}\right\} k^{2}\left[\frac{18 p_{r}\left(1+p_{r}\right) k^{6}}{v^{4}\left(t-t_{1}\right)^{5}\left(1+2 p_{r}\right)}+\right. \\
& \left\{\frac{17+36 p_{r}-2 p_{r}{ }^{2}}{4\left(1+p_{r}\right)^{2} v^{3}\left(t-t_{1}\right)^{4}}+\frac{40 p_{r}\left(1+p_{r}\right)}{3\left(1+2 p_{r}\right) v^{2}\left(t-t_{1}\right)^{3}}-\frac{1}{v^{3}\left(t-t_{1}\right)^{3}}\right\} k^{8}+ \\
& \left\{\frac{17-36 p_{r}+64 p_{r}{ }^{2}-6 p_{r}{ }^{4}}{12\left(1+p_{r}\right)^{3} v^{2}\left(t-t_{1}\right)^{3}}+\frac{14 p_{r}{ }^{2}-104 p_{r}-18}{\left(1+p_{r}\right)^{2} v\left(t-t_{1}\right)}\right\} k^{10} \cdot \exp \left(\omega_{2}\right) \int E i\left(\omega_{2}\right) d t+ \\
& \frac{\left(-\pi^{\frac{1}{2}} \xi_{1} p_{r}^{\frac{9}{2}}\right.}{128 v^{2}\left(1+p_{r}\right)^{\frac{11}{2}}} \exp \left\{\frac{-v\left(t-t_{1}\right)\left(1+2 p_{r}\right)}{p_{r}}\right\} k^{2}\left[\frac{180 p_{r}\left(1+2 p_{r}\right) k^{6}}{v^{4}\left(t-t_{1}\right)^{4}\left(1+2 p_{r}\right)^{6}}+\right. \\
& \left\{\frac{1710-1036 p_{r}-1820 p_{r}^{2}}{4\left(1+p_{r}\right)^{2} v^{3}\left(t-t_{1}\right)^{4}}+\frac{140 p_{r}\left(1+p_{r}\right)}{3\left(1+2 p_{r}\right) v^{2}\left(t-t_{1}\right)^{3}}-\frac{1}{v^{3}\left(t-t_{1}\right)^{3}}\right\} k^{8}+ \\
& \left\{\frac{1267-3226 p_{r}+6234 p_{r}{ }^{2}-146 p_{r}{ }^{4}}{12\left(1+p_{r}\right)^{3} v^{2}\left(t-t_{1}\right)^{2}}+\frac{142 p_{r}{ }^{2}-104 p_{r}-18}{\left(1+p_{r}\right)^{2} v\left(t-t_{1}\right)}\right\} k^{10} \cdot \exp \left(\omega_{3}\right) \int E i\left(\omega_{3}\right) d t
\end{aligned}
$$


$\{$ Where,

$$
\begin{gathered}
\left.E i\left(\omega_{1}\right)=\frac{\exp \left\{\frac{-\left(1+2 p_{r}{ }^{2}\right) t k^{2}}{p_{r}\left(1+p_{r}\right)}\right.}{t-t_{1}}\right\} \text { and, } \omega_{1}=\frac{-\left(1+2 p_{r}{ }^{2}\right) t k^{2}}{p_{r}\left(1+p_{r}\right)}, \operatorname{Ei}\left(\omega_{2}\right)=\frac{\exp \left\{\frac{-v\left(t-t_{1}\right)\left(1-2 p_{r}+2 p_{r}{ }^{2}\right) t k^{2}}{p_{r}\left(1+2 p_{r}\right)}\right.}{t-t_{1}} \text { and } \\
\omega_{2}=\frac{-v\left(t-t_{1}\right)\left(1-2 p_{r}+2 p_{r}{ }^{2}\right) t k^{2}}{p_{r}\left(1+2 p_{r}\right)}, E i\left(\omega_{3}\right)=\frac{\exp \left\{\frac{v\left(1-2 p_{r}\right) t k^{2}}{p_{r}}\right\}}{t-t_{1}} \text { and } \omega_{3}=\frac{v\left(1-2 p_{r}\right) t k^{2}}{p_{r}} \\
T_{a x}
\end{gathered}
$$

Fig-2: universal function for calculating energy spectrum function [see equ. 30]

From equation (29), we get, $T=T_{1}+T_{2}$

$$
\text { where, } \quad T_{1}=J(k) \exp \left[-\frac{2 v k^{2}\left(t-t_{0}\right)}{p_{r}}\right]+T_{\beta} \quad \text { and } \quad T_{2}=T_{\gamma} \text {; }
$$

In equation (33) $T_{1}$ and $T_{2}$ are temperature fluctuation field spectrum arising from consideration of the three and four - point correlation equations respectively. Equation (30) can be integrated over all of all wave numbers to give the total Temperature fluctuation turbulent energy. That is

$$
\left\langle\frac{T \cdot T^{\prime}}{2}\right\rangle=\int_{0}^{\infty} T d k=\int_{0}^{\infty}\left(T_{1}+T_{2}\right) d k
$$

Now, $\quad \int_{0}^{\infty} T_{1} d k=\frac{N_{0} p_{r}^{\frac{3}{2}} v^{-\frac{3}{2}}\left(t-t_{0}\right)^{-\frac{3}{2}}}{8 \sqrt{2 \pi}}+\xi_{0} B_{0} v^{-6}\left(t-t_{0}\right)^{-5}$

And $\quad \int_{0}^{\infty} T_{2} d k=\xi_{1}\left[C_{0} v^{-\frac{9}{2}}\left(t-t_{1}\right)^{-\frac{7}{2}}+D_{0} v^{-\frac{11}{2}}\left(t-t_{1}\right)^{-7}\right]$.

Where, $C_{0}=C_{2}+C_{4}+C_{6}$ and $D_{0}=C_{1}+C_{3}+C_{5}$

$$
\begin{aligned}
& B_{0}=\frac{\pi p_{r}{ }^{6}}{\left(1+p_{r}\right)\left(1+2 p_{r}\right)^{\frac{5}{2}}}\left[\frac{8}{13}+\frac{3 p_{r}\left(1+5 p_{r}\right)}{2\left(1+2 p_{r}\right)}+\ldots . .\right] \\
& C_{1}=\frac{\pi p_{r}{ }^{\frac{7}{2}}}{\left(1+p_{r}\right)^{\frac{5}{2}}\left(1+2 p_{r}-P_{m}{ }^{2}\right)^{\frac{7}{2}}}\left[\frac{13.7}{32}+\frac{13.5\left(13-4 p_{r}+15 p^{2} r_{r}\right.}{2\left(1+2 p_{r}\right)}+\ldots . .\right] \\
& C_{2}=\frac{\pi p_{r}{ }^{\frac{19}{2}}}{\left(1+p_{r}\right)^{\frac{3}{2}}\left(1+2 p_{r}-P_{m}{ }^{2}\right)^{\frac{5}{2}}}\left[\frac{11.7}{32}+\frac{13.7 .5\left(15 p_{r}-14+20 p^{2}\right)}{2^{7}\left(1+2 p_{r}-P_{m}{ }^{2}\right)}+\ldots . .\right]
\end{aligned}
$$




$$
\begin{aligned}
& C_{3}=\frac{\pi p_{r}^{\frac{17}{2}}\left(1+p_{r}\right)^{\frac{1}{2}}}{\left(1+p_{r}\right)^{2}\left(1+2 p_{r}-P_{r}^{2}\right)^{\frac{5}{2}}}\left[\frac{13.7}{32}+\frac{13.5\left(15+28 p_{r}-14 P^{2}+20 p_{r}{ }_{r}\right)}{2^{9}\left(1+p_{r}\right)^{2}\left(1+2 p_{r}-P_{r}^{2}\right)}+\ldots . .\right] \\
& C_{4}=\frac{\pi p_{r}{ }^{\frac{21}{2}}}{\left(1+p_{r}\right)^{\frac{3}{2}}\left(1+2 p_{r}-P_{m}{ }^{2}\right)^{\frac{7}{2}}}\left[\frac{11.7 .3}{16}+\frac{13.7 .5\left(-20 p_{r}-17 P_{r}^{2}+20 p_{r}{ }^{3}\right)}{2^{7}\left(1+2 p_{r}-P_{m}{ }^{2}\right)}+\ldots . .\right] \\
& C_{5}=\frac{\pi p_{r} r^{\frac{17}{2}}\left(1+p_{r}\right)^{\frac{1}{2}}}{\left(1+p_{r}\right)^{\frac{17}{2}}\left(1+2 p_{r}\right)^{\frac{7}{2}}}\left[\frac{13.7 .5 .3}{32}-\frac{13.5 .3\left(15+28 p_{r}-14 P_{r}^{3}+20 p_{r}^{5}\right)}{2^{11}\left(1+p_{r}\right)^{2}}+\ldots . .\right] \\
& C_{6}=\frac{\pi p_{r}^{\frac{21}{2}}}{\left(1+p_{r}\right)^{\frac{11}{2}}\left(1+2 p_{r}\right)^{\frac{9}{2}}}\left[\frac{13.11 .7 .3}{16}-\frac{11.9 .7 .5\left(20+20 p_{r}-17 P_{r}^{2}+20 p_{r}{ }^{3}\right)}{2^{7}\left(1+2 p_{r}-P_{m}{ }^{2}\right)}+\ldots . .\right] \\
& \frac{\left\langle T . T^{\prime}\right\rangle}{2}=\frac{N_{0} p^{\frac{3}{2}} r v^{-\frac{3}{2}}\left(t-t_{0}\right)^{-\frac{3}{2}}}{8 \sqrt{2 \pi}}+\xi_{0} X v^{-6}\left(t-t_{0}\right)^{-5}+\xi_{1}\left[R v^{-\frac{9}{2}}\left(t-t_{1}\right)^{-\frac{7}{2}}+S v^{-\frac{11}{2}}\left(t-t_{1}\right)^{-7}\right] .
\end{aligned}
$$

Also, we can write the equation (34) of the following form,

$$
\left\langle T^{2}\right\rangle=W\left(t-t_{0}\right)^{-3 / 2}+X\left(t-t_{0}\right)^{-5}+Y\left(t-t_{1}\right)^{-\frac{7}{2}}+Z\left(t-t_{1}\right)^{-7} \text {, }
$$

This is the decay of energy of Temperature fluctuation in homogeneous turbulence for four point correlations.

Where, $\left\langle T^{2}\right\rangle=\left\langle T \cdot T^{\prime}\right\rangle, W=\frac{N_{0} p_{r}^{\frac{3}{2} \nu^{-\frac{3}{2}}}}{4 \sqrt{2 \pi}} X=2 \xi_{0} X v^{-6}, Y=2 \xi_{1} R v^{-\frac{9}{2}}$ and $Z=2 \xi_{1} S v^{-\frac{11}{2}}$

If $\mathrm{R}=0$ and $\mathrm{S}=0$ that is $\mathrm{Y}=0$ and $\mathrm{Z}=0$ in equation (38), it will be in the form

$$
\left\langle T^{2}\right\rangle=A\left(t-t_{0}\right)^{-3 / 2}+B\left(t-t_{0}\right)^{-5}, \quad \text { taking } \mathrm{W}=\mathrm{A}, \mathrm{Y}=\mathrm{B}
$$

which is completely same with the result of A. L. Loeffler and R. G, Deissler [ 6 ] for the case of three -point correlation. In equation (38), the third and fourth term on the right hand side comes due to four point correlations.

\section{Result and discussions-}

The evaluation of analytical results reported in this paper is performed and representative set of results is reported graphically. These results are obtained to illustrate the influence of various parameters on the temperature fluctuations. For numerical validation of the analytical results, we have taken the results obtained in equations (38) and (40). The constants W, X, Y, Z, is calculated in terms of Prandtl no.p. $\mathrm{r}_{\mathrm{r}}$ constants $\mathrm{N}_{0}, \xi_{0}, \xi_{1}$, kinematic viscosity $v$, thermal conductivity $\mathrm{k}$. In the present study we adopted the following default parametric values for some fluid in the table has discussed step by step. When the Prandtl No. is small such as of mercury $\mathrm{p}_{\mathrm{r}}=.015$ then from (39)

Table-1:The value of the constants and parameter used in equation (38)and (40).

\begin{tabular}{|l|l|l|l|l|l|l|l|l|l|}
\hline Fluid & $\mathrm{P}_{\mathrm{r}}$ & $\boldsymbol{V}$ & $\mathrm{N}_{0}$ & $\xi_{0}$ & $\xi_{1}$ & $\mathrm{~W}$ & $\mathrm{X}$ & $\mathrm{Y}$ & $\mathrm{Z}$ \\
\hline \multirow{2}{*}{ Mercury } & 0.015 & 0.10 & .1 & .01 & .02 & .00058 & $4.18 \times 10^{-7}$ & $3.69 \times 10^{-13}$ & 5.87 \\
\cline { 2 - 10 } & 0.015 & 0.08 & .1 & .01 & .02 & .00081 & $1.6 \times 10^{-6}$ & $-1.01 \times 10^{-12}$ & 20.03 \\
\hline \multirow{2}{*}{ Mix Gas } & 0.2 & 80 & .1 & .01 & .02 & $1.15 \times 10^{-6}$ & $5.75 \times 10^{-18}$ & $3.78 \times 10^{-16}$ & $9.95 \times 10^{-13}$ \\
& 0.2 & 200 & .1 & .01 & .02 & $3.15 \times 10^{-7}$ & $2.36 \times 10^{-20}$ & $6.12 \times 10^{-18}$ & $6.44 \times 10^{-15}$ \\
\hline \multirow{2}{*}{ Hyd Gas } & .04 & 100 & .1 & .01 & .02 & $2.5 \times 10^{-6}$ & $6.8 \times 10^{-17}$ & $2.7 \times 10^{-14}$ & $9.79 \times 10^{-13}$ \\
\cline { 2 - 9 } & 0.4 & 300 & .1 & .01 & .02 & $4.86 \times 10^{-7}$ & $9.4 \times 10^{-20}$ & $1.9 \times 10^{-16}$ & $2.3 \times 10^{-15}$ \\
\hline \multirow{2}{*}{ Hel Gas } & 0.7 & 120 & .1 & .01 & .02 & $4.6 \times 10^{-6}$ & $4.8 \times 10^{-16}$ & $7.4 \times 10^{-13}$ & $9.4 \times 10^{-23}$ \\
& 0.7 & 400 & .1 & .01 & .02 & $7.6 \times 10^{-7}$ & $3.4 \times 10^{-19}$ & $3.3 \times 10^{-15}$ & $1.2 \times 10^{-15}$ \\
\hline
\end{tabular}




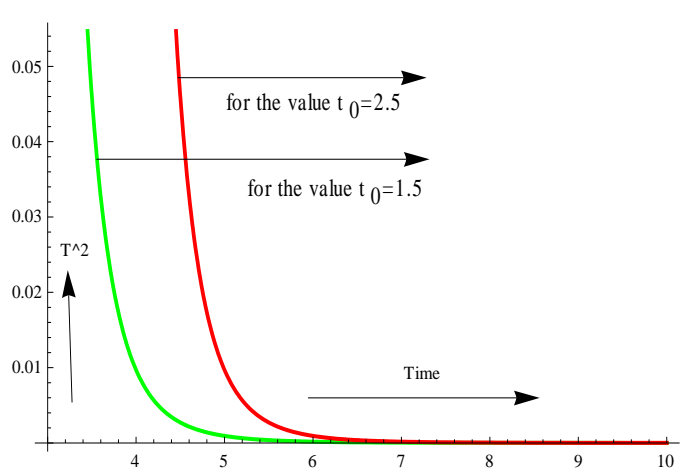

Figure-1(a)

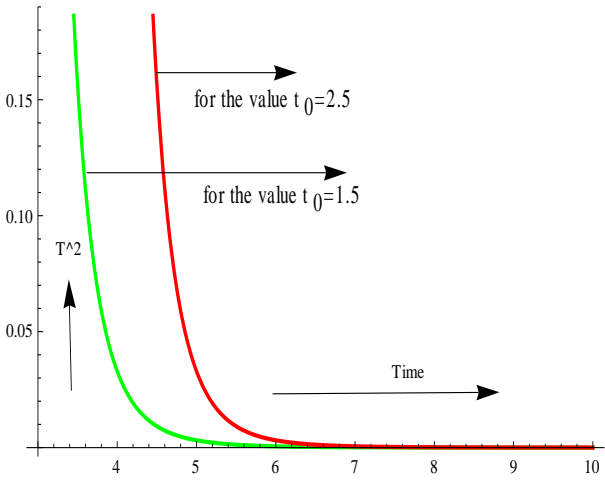

Figure-1(b)

Figure-1(a), Figure-1(b) represents the energy decay of temperature fluctuation for four -point correlations of equation (38). When the Prandtl No. is small as of mercury $\mathrm{P}_{\mathrm{r}}=.015$ and $v=0.1$ at $20^{\circ} \mathrm{C}$, Temp $v=0.08$ at $80^{\circ} \mathrm{C}$ Temp. It is observed that the energy decays more rapidly as viscosity decreases from 0.1 to 0.08 at temp. $20^{\circ}$ to $80^{\circ} \mathrm{C}$.

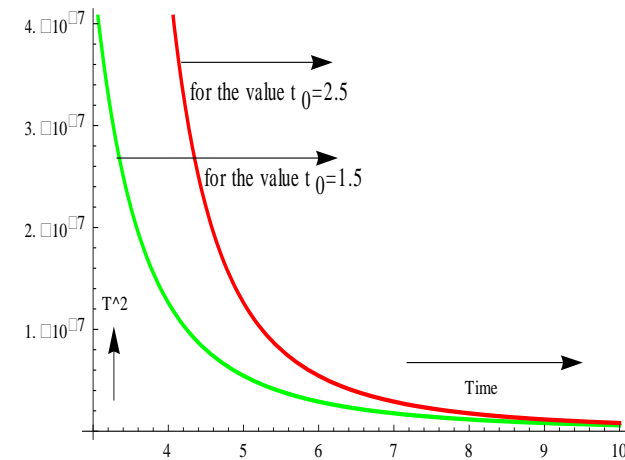

Figure-2(a): Energy decay for mix. of gas $\mathrm{p}_{\mathrm{r}}=.2$ and $v=80$ at $20^{\circ} \mathrm{C}$ Temp

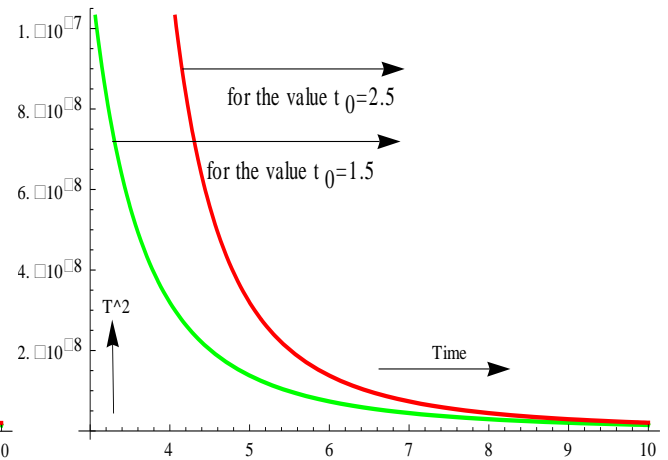

Figure-2(b): Energy decay for mix. of gas $\mathrm{p}_{\mathrm{r}}=.2$ and $\boldsymbol{v}=80$ at $20^{\circ} \mathrm{C}$ Temp

Figure-2(a) and Figure-2(b) represent the energy decay of temperature fluctuation for four -point correlations of equation (38). When the Prandtl No. is as of mix. of gas $\mathrm{P}_{\mathrm{r}}=.2$ and $\nu=80$ at $20^{\circ} \mathrm{C}$ Temp, $\nu=200$ at $80^{\circ} \mathrm{C}$ Temp In this case, energy decays too much rapidly as viscosity decreases from 200 to 80 at the same pr. no.

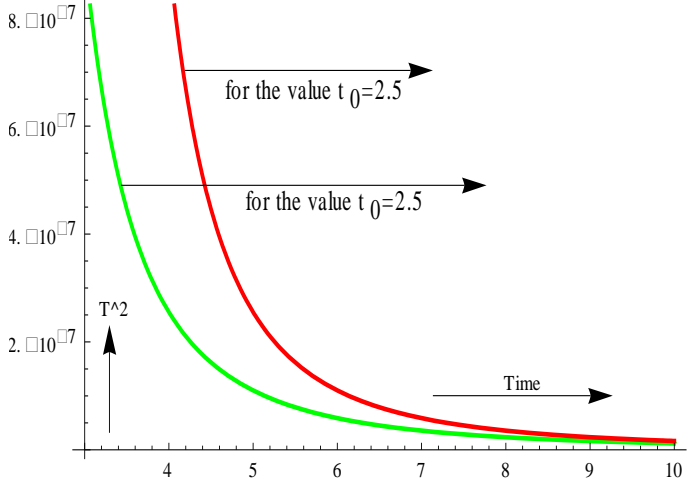

Figure-3(a)

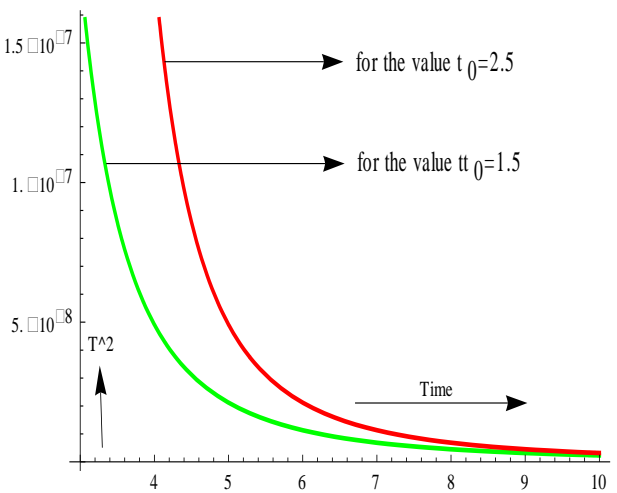

Figure-3(b)

Figure-3(a), Figure-3(b) represents the energy decay of temperature fluctuation for four -point correlations of equation (38). When the Prandtl No. is 1 as of Hyd gas $\mathrm{P}_{\mathrm{r}}=4$ and $v=100$ at $0^{\circ} \mathrm{C}$ Temp $v=300$ at $100^{\circ} \mathrm{C}$ Temp Result: Energy decay rapidly as viscosity decries 300 to 100

Comparing fig 1, 2 and 3: we see that Energy decay rapidly more and more about $10^{5}$ times as Prandtl No. decreases in Fig1 and Fig2 if it is used mix. gas than mercury. That means energy decays 10 million times for mix. gas from mercury at the same time range. On the other hand, in fig-3 we use hyd. Gas instead of mercury. Prandtl no. for mercury is 0.015 and for hydrogen 0.4 that we have used in fig-1 and fig-3 restively .We 
observes that energy decays $2 \times 10^{5}$ times as Prandtl no. decreases for mercury than from hyd. gas. For this reason, Hydrogen gas is to be used in different jet engines.
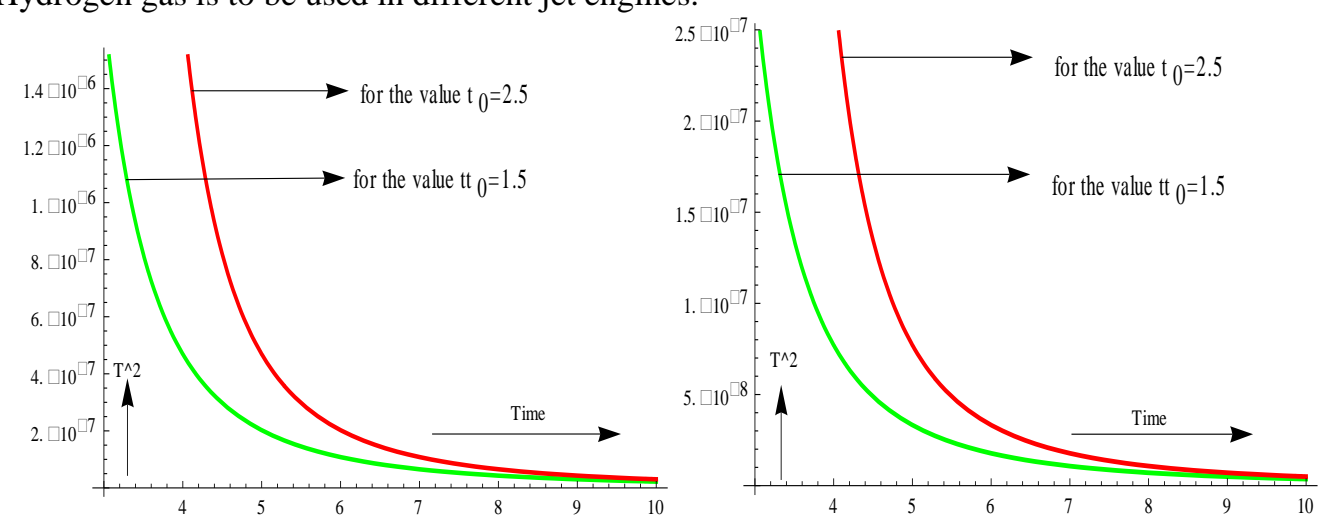

Figure-4 represents the energy decay of temperature fluctuation for four -point correlations of equation (38). When the Prandtl No. is 1 as of Hel gas $\mathrm{P}_{\mathrm{r}}=.7$ and $\nu=120$ at $0^{0} \mathrm{C}$ Temp Figure-4' represents the energy decay of temperature fluctuation for four-point correlations of equation (38). When the Prandtl No. is 1 as of

Hyd gas $\mathrm{P}_{\mathrm{r}}=.4$ and $v=400$ at $100^{\circ} \mathrm{C}$ Temp Energy decay rapidly as viscosity decries from 400 to 120 Comparing fig -1 and 2, fig- 3 , and 4 we see that Energy decay about $10^{5}, 5 \times 10^{4}$, times as Prandt No. decreases

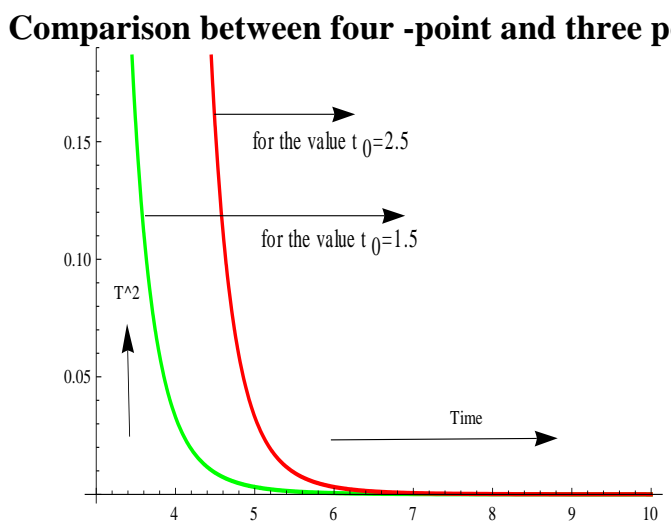

Fig 5(a): Energy decay curves of equation. (38)

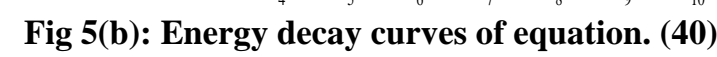

Fig-5(a) and Fig 5(b)represents the energy decay of temperature fluctuation for four -point and three point correlations of equation (38) and (40). When the Prandtl No. is small as of mercury $\mathrm{P}_{\mathrm{r}}=.015$ and $v=0.08$ at $80^{\circ}$ $\mathrm{C}$ Temp. It is clear that, in four point correlations energy decays more rapidly than three point correlations.

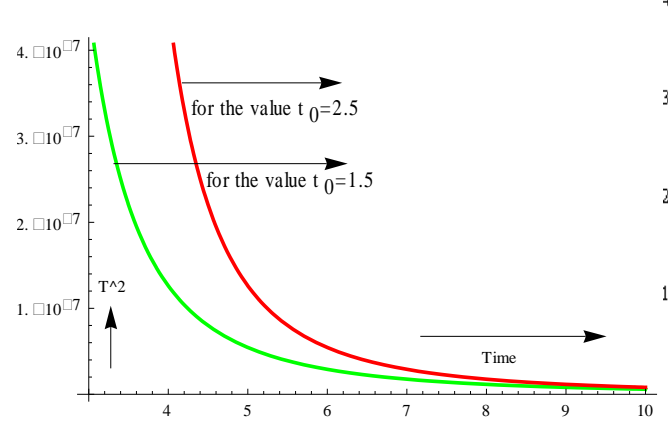

Fig 6(a) Four point equ. (38)

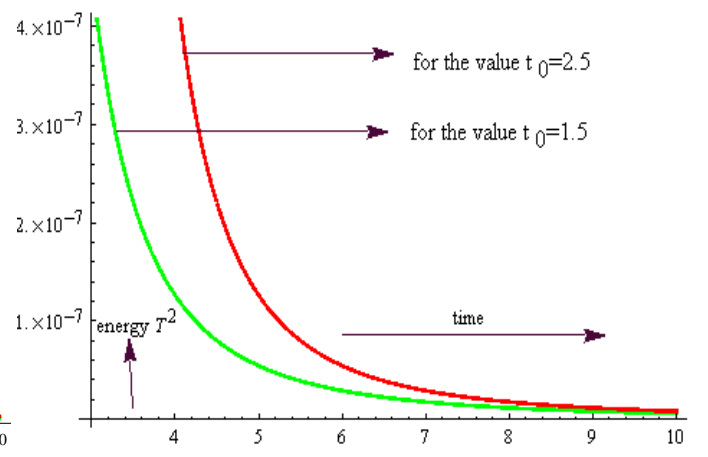

Fig 6(b) three point equ. (40)

Fig-6(a) and Fig 6(b) represents the energy decay of temperature fluctuation for four -point and three point correlations of equation (38) and (40). When the Prandtl No. is small as of mix. gas $\mathrm{P}_{\mathrm{r}}=.2$ and $\nu=80$ at $0^{0} \mathrm{C}$ Temp.

In this case, there is no change in energy decay at same viscosity and Prandtl No. 


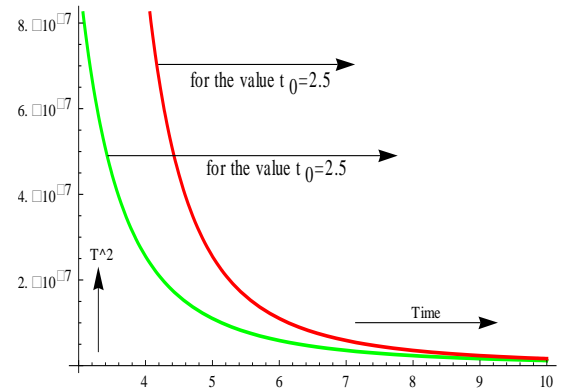

Fig 7(a) Four point equ. (38)

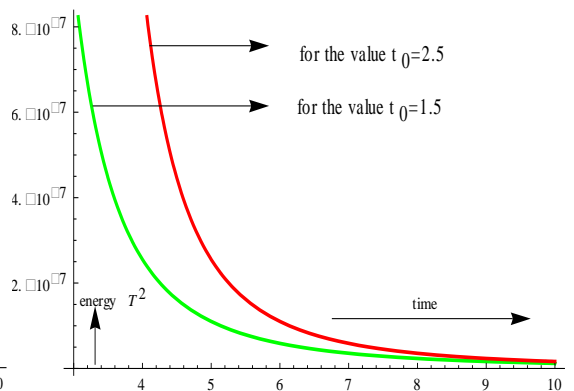

Fig 7(b) three point equ. (40)

Fig-7(a) and Fig-7(b) represents the energy decay of temperature fluctuation for four -point and three point correlations of equation (38) and (40). When the Prandtl No. is small as of hyd. gas $\mathrm{P}_{\mathrm{r}}=.4$ and $\nu=100$ at $0^{0} \mathrm{C}$ Temp. We observed that there is no change in energy decays for four point and three point correlations as for same viscosity .

\section{Conclusions}

Through this study the result is obtain by neglecting quintuple correlation the four point correlation equations appear to represent the homogeneous turbulence for times between the initial and final period in temperature fluctuations. If the quintuple correlations were considered in this study, it appears more terms in higher power of $\left(\mathrm{t}-\mathrm{t}_{0}\right)$ would be added to the equation (38). The terms for higher order correlation in Decay law die out faster than those for lower order ones, in agreement with the fundamental assumption made in the analysis.

In equation (38), it is observed that the temperature fluctuation turbulent energy for four- point correlations systems decays rapidly more and more by exponential manner than the decays of three point correlation system. .

\section{Acknowledgement}

This work is supported by the research grant under the 'University grant Commission (UGC), Bangladesh and the authors (a malek) acknowledge the support. The authors also express their gratitude to the Department of Applied Mathematics, University of Rajshahi for providing us all facilities regarding this article.

\section{References}

[1]. G. I. Taylor, Statistical theory of turbulence. Proc. Roy. Soc. London. A 151 (1935), 421-454.

[2]. S. Chandrasekhar, The invariant theory of isotropic turbulence in magneto-hydrodynamics, Proc. Roy. Soc., London, A204, (1951a), 435-449.

[3]. S. Corrsin, on spectrum of isotropic temperature fluctuations in isotropic turbulence, J. Apll. Phys, 22(1951b), 469-473.

[4]. R. G. Deissler, on the decay of homogeneous turbulence before the final period, Phys .Fluids 1(1958), 111-121.

[5]. R. G. Deissler, A theory of Decaying Homogeneous turbulence, Phys. Fluids 3(1960), 176-187.

[6]. A. L. Loeffler and R.G. Deissler, Decay of temperature fluctuations in homogeneous turbulence before the final period, Int. J. Heat Mass Transfer, 1(1961), 312-324.

[7]. M. S. A. Sarker and M. A. K. Azad, Decay of temperature fluctuations in homogeneous turbulence before the final period for the case of multi-point and multi-time in a rotating system, Bangladesh J. Sci. Ind. Res., 41, (3-4), 147-158, 2006.

[8]. M. A. K. Azad and M.S. Alam Sarker, Decay of temperature fluctuations in homogeneous turbulence before the final period for the case of multi-point and multi-time in presence of dust particle, Rajshahi University Studies, Part-B, Journal of Science, 34, 37-50, 2006

[9]. M. A. K. Azad and M.S. Alam Sarker, Decay of temperature fluctuations in homogeneous turbulence before the final period for the case of multi-point and multi-time in a rotating system in presence of dust particle, Journal of Applied Sciences Research, 4(7),793-802,2008

[10]. M. A. K. Azad and M.S. Alam Sarker, Decay of temperature fluctuations in MHD turbulence before the final period in a rotating system, Journal of Bangladesh J. Sci. Ind. Res,44(4), 407-414,2009

[11]. M. A. K. Azad, M. S. Alam Sarker and M. A. Aziz, Decay Law of temperature fluctuations in dusty fluid MHD Turbulence before the final Period, J. Math. Forum, X, 32-48, 2007-7008, 2009.

[12]. M. H. U. Molla, M. A. K. Azad and M. Z. Rahman, Decay of Temperature Fluctuations in homogeneousTurbulenc Before the Finaln Period in a Rotating System, Research Journal Mathematics and Statistics(RJMS), 4(2) , 45-51, 2012

[13]. M. A. Bkar PK, M. A. K. Azad and M.S .Alam Sarker, Decay of Energy of MHD Turbulence For Four-point Correlation, International Journal of Engineering Research \& Technology (IJERT) , 1 (9), 1 -13, 2012

[14]. M. A. K. Azad and Mist. Mumtahinah, Decay of Temperature fluctuations in dusty fluid homogeneous turbulence prior to final period, Research Journal of Applied Sciences, Engineering and Technology 6(8): 1490-1496, 2013

[15]. M. A. Bkar PK, M. S. A. Sarker and M. A. K. Azad, Homogeneous Turbulence in a First-order Reactant for the Case of Multipoint and Multi-time Prior to the Final Period of Decay in a Rotating System, Research Journal of Applied Sciences, Engineering and Technology 6(10): 1749-1756, 2013

[16]. M. A. K. Azad and Mist. Mumtahinah, Decay of Temperature fluctuations in dusty fluid homogeneous turbulence prior to the ultimate period in presence of coriolis force, Res. J. Appl. Sci. Eng. and Tech. 7 (10), 1932-1939, 2014. 